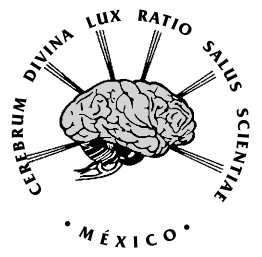

\title{
Evaluación neuropsicológica y aprendizaje de las matemáticas en educación básica
}

\author{
Neuropsychological assessment and learning mathematics in basic \\ education
}

\author{
Dora Elizabeth Granados Ramos*
}

* Universidad Veracruzana, Facultad de Psicología-Xalapa, México. Laboratorio de Psicobiología.

\begin{abstract}
RESUMEN
Palabras clave: El objetivo de la presente investigación consistió en describir la relación entre las nociones matemáticas y los procesos cognoscitivos Neuropsicología, visuales de memoria, percepción, atención y las habilidades espaciales en niños de quinto y sexto grado de educación primaria. matemáticas, Se realizó una investigación descriptiva, observacional, transversal y prospectiva con 42 niños de quinto y sexto grado de escuelas desarrollo, oficiales de Xalapa, Veracruz: 21 niños y 21 niñas, con edad promedio de 10 años 9 meses (DE \pm 10 meses). Se aplicó la evaluación aprendizaje. neuropsicológica infantil (ENI) para evaluar las nociones matemáticas, percepción, atención, memoria y habilidades espaciales, y la escala de inteligencia Wechsler (WISC-IV) para obtener el coeficiente intelectual. El desempeño mayor correspondió a las subescalas de razonamiento con el $71 \%$ y a conteo con el $69 \%$ de los niños que obtuvieron puntajes más altos. En los procesos cognoscitivos evaluados tuvieron puntajes altos: el $93 \%$ en percepción visual, el $74 \%$ en memoria visual y el $64 \%$ en habilidades espaciales. En atención visual, el $55 \%$ obtuvo puntajes bajos. Se observaron diferencias significativas con una $p<.05$ al comparar manejo numérico con atención visual y con habilidades espaciales; también al comparar conteo con habilidades espaciales y cálculo con atención visual.
\end{abstract}

\section{ABSTRACT}

Key words: The aim of this investigation was to describe the relationship between mathematical concepts and cognitive processes of Neuropsychology, mathematics, development, visual memory, perception, attention and spatial skills in children in fifth and sixth grade of primary education. A descriptive, observational, cross-sectional and prospective study with 42 children in fifth and sixth grade of official schools from Xalapa, Veracruz was performed: 21 boys and 21 girls, mean age ten years nine months (SD \pm 10 months). Child neuropsychological assessment (ENI) was applied to evaluate mathematical concepts, perception, attention, memory, spatial skills and Wechsler Intelligence Scale (WISC-IV) for the IQ. The higher performance corresponded to the subscales of reasoning with $71 \%$ and counting with $69 \%$ of children scored higher. In the cognitive processes assessed, had high scores: $93 \%$ in visual perception, $74 \%$ in visual memory and $64 \%$ in spatial skills. In visual attention $55 \%$ scored low scores. Significant differences at $p<.05$ were observed, when comparing numerical management with visual attention and spatial skills; also comparing count with spatial skills and calculation with visual attention.

\section{PLANTEAMIENTO DEL PROBLEMA}

En la educación básica se observan con frecuencia alumnos con dificultad para el aprendizaje básico de la lectoescritura y las matemáticas.

Las dificultades en el aprendizaje de las matemáticas, objeto de estudio de esta investigación, se han documentado en diferentes países y niveles educativos.
Estos problemas pueden deberse a causas biológicas, psicológicas o ambientales que influyeron en la etapa pre, peri o postnatal y que se observan en las etapas formales educativas cuando los niños se enfrentan al manejo del número, la representación de cantidades, las operaciones con números, la resolución de problemas y las representaciones geométricas. 
Ante las dificultades en matemáticas descritas, es necesario determinar qué problemas se presentan, por qué en algunos casos persisten y cómo podrían resolverse o evitarse en el futuro con estrategias de enseñanza o terapéuticas adecuadas.

Se ha documentado que en el nivel universitario algunos alumnos eligen estudiar carreras profesionales que no impliquen el estudio de las matemáticas, debido a que las rechazan o tienen aversión por las experiencias de años escolares previos. ${ }^{1}$

En muchos casos se asocia el nivel de inteligencia con el éxito en el aprendizaje de las matemáticas, por lo que en este estudio se analiza el desempeño en tareas matemáticas de niños con inteligencia promedio y actividad eléctrica cerebral normal, con objeto de conocer la relación entre procesos visuales de percepción, atención, memoria y habilidades espaciales y el desempeño en el aprendizaje de las matemáticas de niños de educación básica.

\section{INTRODUCCIÓN}

La neuropsicología es una disciplina en la que interactúan diversos campos de estudio como son la neurología, la psicología, la neuroanatomía, la neurofisiología y la neuroquímica. Esta disciplina ha permitido explicar procesos cognoscitivos como la atención, la percepción, el lenguaje y la capacidad de resolver problemas en condiciones normales o patológicas.

A través de la neuropsicología se han descrito los aprendizajes escolares de lecto-escritura y matemáticas en el desarrollo normal y ante trastornos del aprendizaje. ${ }^{2}$ Específicamente, en el aprendizaje de las matemáticas es importante conocer los procesos neuropsicológicos implicados en el cálculo, manejo de operaciones básicas como la adición, la sustracción, la multiplicación, la división y el estudio de las reglas del razonamiento matemático para dar solución a las dificultades que puedan presentarse en su adquisición.

Se ha descrito que el conocimiento matemático es un conjunto de medios específicos y disponibles que le permiten al niño solucionar problemas y llevar a cabo con éxito las tareas de la vida cotidiana. ${ }^{3}$

De acuerdo a Piaget, ${ }^{4}$ las bases de las nociones lógico-matemáticas se estructuran desde etapas tempranas cuando el niño interactúa con los objetos que hay en su medio ambiente y en la etapa preoperatoria el niño logra representar y verbalizar a través de símbolos o números los objetos que hay en su contexto. El autor describe dicha evolución a partir de cuatro periodos en el desarrollo a través de los cuales se logra pasar de conocimientos concretos a abstractos: el sensorio-motor o de inteligencia preverbal (0-2 años), el preoperatorio (2-7 años), el de las operaciones concretas (7-11 años) y el de las operaciones formales (a partir de los 11 o 12 años en adelante).

Entre los 6 y 7 años, aproximadamente, época en la que los niños se encuentran en la primaria, inicia el aprendizaje formal de las matemáticas; sus acciones sucesivas los conducen a realizar operaciones a partir de clasifica- ciones, seriaciones y correspondencias que se construyen simultáneamente. La base de dicha construcción se sustenta en la interacción con los objetos y en descubrir las características de los mismos como color, tamaño, forma, ubicación en el espacio y en el tiempo. ${ }^{3,5}$ De los 11 a los 12 años, etapa del pensamiento formal e inicio de la adolescencia, se elaboran agrupaciones más complejas que caracterizan a la inteligencia reflexiva completa. ${ }^{3}$

En el desarrollo de las nociones matemáticas tienen lugar procesos cognoscitivos básicos como la memoria y la atención. La atención es un proceso de alertamiento, activación generalizada, acción selectiva y concentración; ${ }^{6}$ es un mecanismo central de control del procesamiento de información que actúa de acuerdo con los estímulos que se atienden en un momento determinado, siendo necesario dejar de dar respuesta a aquello que es irrelevante a la tarea que se está realizando. ${ }^{7}$ La memoria es un prerrequisito para toda actividad cognoscitiva; es la encargada de registrar, almacenar y recuperar la información captada por medio de los sentidos. ${ }^{8}$

Se ha sustentado que en el aprendizaje de las matemáticas son relevantes los procesos de discriminación visual, memoria visual y relaciones visuoespaciales que proveen significado a los datos. ${ }^{9}$

Las competencias matemáticas iniciales van más allá de la adquisición de los principios subyacentes al conteo y la habilidad para determinar la cardinalidad de un conjunto y la ordinalidad de diversos objetos o sucesos; implican pasar de la coordinación de acciones a la reversibilidad, de la capacidad de establecer relaciones entre las cantidades a la de realizar operaciones diversas. ${ }^{10}$

En la adquisición y el manejo de la convencionalidad de los sistemas numéricos se requiere del desarrollo adecuado de la percepción, la atención y la memoria. Es necesario que los niños reciban una enseñanza formal que tome en cuenta las experiencias previas tanto del medio familiar como preescolar y que les permita conocer los símbolos, la sintaxis y la semántica propia de cada uno de ellos. ${ }^{3}$

En el proceso de aprendizaje de las nociones matemáticas pueden presentarse dificultades debido a causas neurobiológicas o neuropsicológicas, como en los casos con discalculia en los que se encuentran alteraciones en el surco intraparietal, que se expresan como dificultades tempranas en el desarrollo numérico, inversiones de números, la inadecuada colocación de los números en las operaciones, problemas de cálculo y en geometría. ${ }^{11,12}$

Se ha descrito que del 3 al $8 \%$ de los alumnos de educación básica presentan dificultades en el aprendizaje de las matemáticas. ${ }^{13}$ Otro tipo de dificultades se relaciona con causas ambientales o psicológicas en cuanto a la ansiedad y la fobia hacia las matemáticas como consecuencia de métodos de enseñanza indadecuados. ${ }^{14}$

Los problemas en matemáticas pueden expresarse como dificultad para comprender y seguir instrucciones o recordar hechos numéricos, o bien errores de cálculo o de procedimiento al intentar resolver problemas; también dificultades visuoespaciales para representar, interpretar y comprender la información. ${ }^{15}$ 
El objetivo de la presente investigación consistió en describir la relación entre las nociones matemáticas y los procesos cognoscitivos visuales de memoria, percepción, atención y las habilidades espaciales en niños de quinto y sexto grado de educación primaria.

\section{MATERIAL Y MÉTODOS}

Se realizó una investigación observacional, transversal y prospectiva.

Se documentaron las variables de grado escolar, sexo, edad, coeficiente intelectual, nociones matemáticas con sus subescalas de conteo, manejo numérico, cálculo y razonamiento; los procesos cognoscitivos visuales de percepción, atención y memoria y las habilidades espaciales.

La muestra se conformó con 42 niños de quinto y sexto grado de escuelas oficiales de Xalapa, Veracruz; 21 niños y 21 niñas, con edad promedio de 10 años 9 meses (DE \pm 10 meses), con electroencefalograma normal (EEG) y coeficiente intelectual $(\mathrm{Cl})$ promedio de 90 (DE \pm 10.8$)$.

Se aplicó la evaluación neuropsicológica infantil $(E N I)^{16}$ para determinar las nociones matemáticas, los procesos cognoscitivos visuales de percepción, atención, memoria y habilidades espaciales y la escala de inteligencia Wechsler (WISC-IV) ${ }^{17}$ para obtener el coeficiente intelectual $(\mathrm{Cl})$.

Las subpruebas de la ENI permiten determinar la presencia de cambios cognoscitivos y comportamentales de niños entre 6 y 15 años de edad. De esta evaluación se tomaron los percentiles de las subpruebas de nociones matemáticas: conteo, manejo numérico, cálculo y razonamiento; los procesos cognoscitivos visuales de percepción, atención y memoria, y de habilidades espaciales. Se calificaron de acuerdo a los criterios de la ENI como puntajes extremadamente bajos $\leq 2$; bajos de 3 a 10; promedio bajo de 11 a 25; promedio medio de 26 a 75 , y por arriba del promedio $>75$ alto. En términos generales, se agruparon como puntajes bajos los de $\leq 2$ a 26 y como puntajes altos los de $27 \mathrm{a} \geq 75$.

El coeficiente intelectual se documentó con la escala de inteligencia WISC-IV para población mexicana, la cual permite medir las habilidades generales de pensamiento y razonamiento de niños entre 6 y 16 años de edad. Se anotó el índice compuesto de la escala total y se clasificó como lo señalan los criterios de evaluación: < 69 muy bajo, 70-79 límite, 80-89 promedio bajo, 90-109 promedio, 110119 promedio alto, $120-129$ superior y > 130 muy superior. Todos los niños tenían EEG normal en el momento de las evaluaciones.

El análisis de las variables se realizó a través del programa JMP 8 en donde se obtuvieron las medidas de tendencia central y se aplicó una prueba no paramétrica de Wilcoxon al comparar las nociones matemáticas: conteo, manejo numérico, cálculo y razonamiento, con los procesos cognoscitivos visuales de percepción, atención y memoria y las habilidades espaciales. Se realizó una correlación de Pearson para comparar las subpruebas de nociones matemáticas con el sexo.

\section{RESULTADOS}

Al analizar los resultados obtenidos en las nociones matemáticas se observó que el desempeño mayor correspondió a las subescalas de razonamiento con el $71 \%$ y a conteo con el $69 \%$, mientras que el desempeño menor correspondió a las áreas de cálculo con el $48 \%$ y a manejo numérico con el $43 \%$.

En cuanto a los procesos cognoscitivos se observó que el $93 \%$ de los niños tuvo puntajes altos en percepción visual, el $74 \%$ en memoria visual y el $64 \%$ en habilidades espaciales. Sin embargo, en atención visual el $55 \%$ obtuvo puntajes bajos.

No se encontraron diferencias significativas al comparar las nociones matemáticas con la percepción visual ni con el sexo. Tampoco se encontraron diferencias significativas al comparar las subpruebas de razonamiento con los procesos cognoscitivos visuales.

Se observaron diferencias significativas al comparar el manejo numérico con la memoria visual (g.l. = 1,40; $p<$ 0.02 ) donde los casos con puntajes bajos en memoria visual correspondieron a los puntajes menores de manejo numérico (Figura 1).

Se encontraron diferencias significativas al comparar las habilidades espaciales con el manejo numérico (g.l. = 1.40; $p<0.03$ ) y el conteo (g.l. = 1.40; $p<0.04$ ), observándose puntajes bajos en el $43 \%$ de manejo numérico, el $36 \%$ de habilidades espaciales y el $31 \%$ de conteo (Figura 2).

Se observaron diferencias significativas al comparar el cálculo con la atención visual (g.l. = 1.40; $p<0.03$ ), donde correspondieron los puntajes bajos de atención visual a los puntajes bajos de cálculo (Figura 3).

\section{DISCUSIÓN}

En esta investigación resaltamos la importancia de las nociones matemáticas para la solución de problemas y la ejecución con éxito en las tareas de la vida cotidiana, así como la importancia de conocer el desempeño que tienen los niños en su educación básica, ya que en esta etapa se establecen las bases para realizar operaciones y razonamientos más complejos.

En las evaluaciones neuropsicológicas de los niños de quinto y sexto grado encontramos que los procesos cognoscitivos de atención y memoria visual se relacionaron con las subpruebas de manejo numérico y de cálculo. Es importante revisar si en los programas educativos de educación básica se favorece el desarrollo de procesos cognoscitivos básicos, ya que la memoria y la atención están íntimamente relacionadas con los aprendizajes escolares.

En la subprueba del manejo numérico confirmamos que la atención y la memoria visual son procesos básicos para el aprendizaje de las nociones matemáticas ${ }^{8,6}$ en cuanto a la capacidad para leer, escribir, comparar y ordenar números, así como para resolver mentalmente y por escrito problemas aritméticos.

De la misma forma, para el manejo numérico y el conteo resultó relevante el dominio de las habilidades espa- 


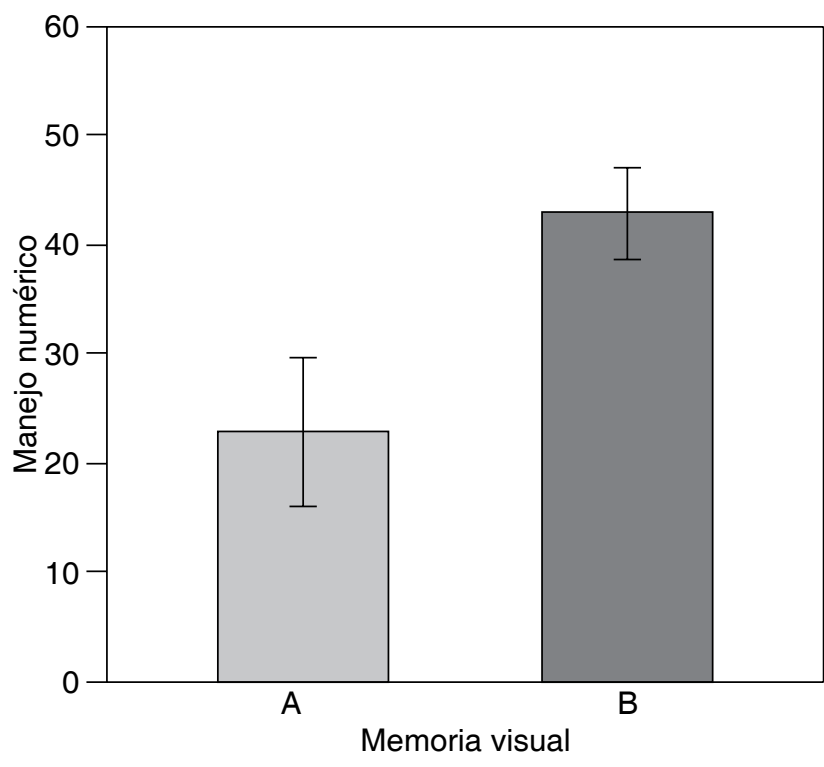

Figura 1. Comparación entre los puntajes de manejo numérico y memoria visual. A corresponde a los puntajes bajos y $\mathrm{B}$ a los puntajes altos.

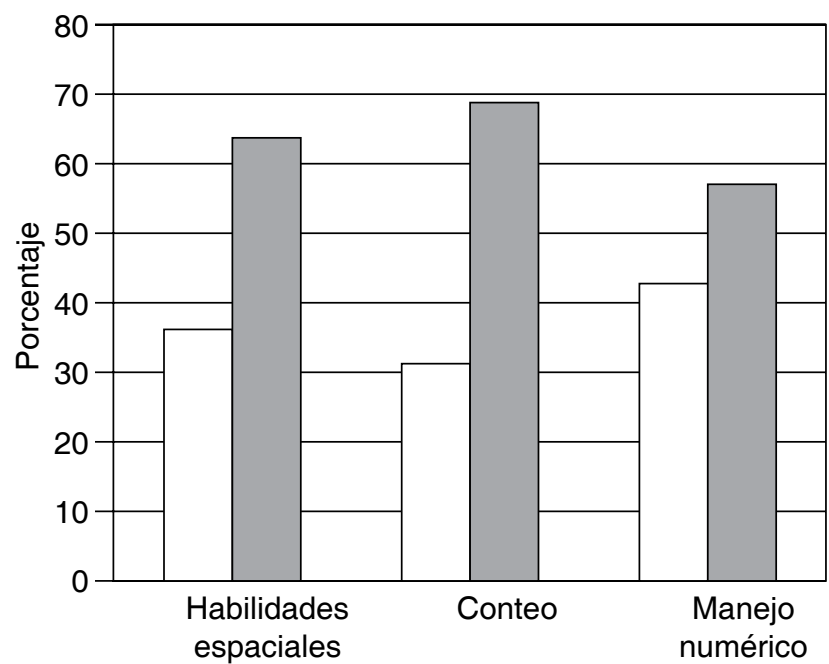

Puntajes bajos $\square$ Puntajes altos

Figura 2. Porcentaje de casos con puntajes bajos y altos en habilidades espaciales, conteo y manejo numérico.

ciales ya que es relevante el dominio del espacio para operar con unidades, decenas y centenas a partir de la representación de los números en la resolución de operaciones básicas.

Si la metodología de la enseñaza de las matemáticas favorece el razonamiento y la comprensión para la solución de los problemas cotidianos, contribuirá al desarrollo del pensamiento matemático desde la educación básica,

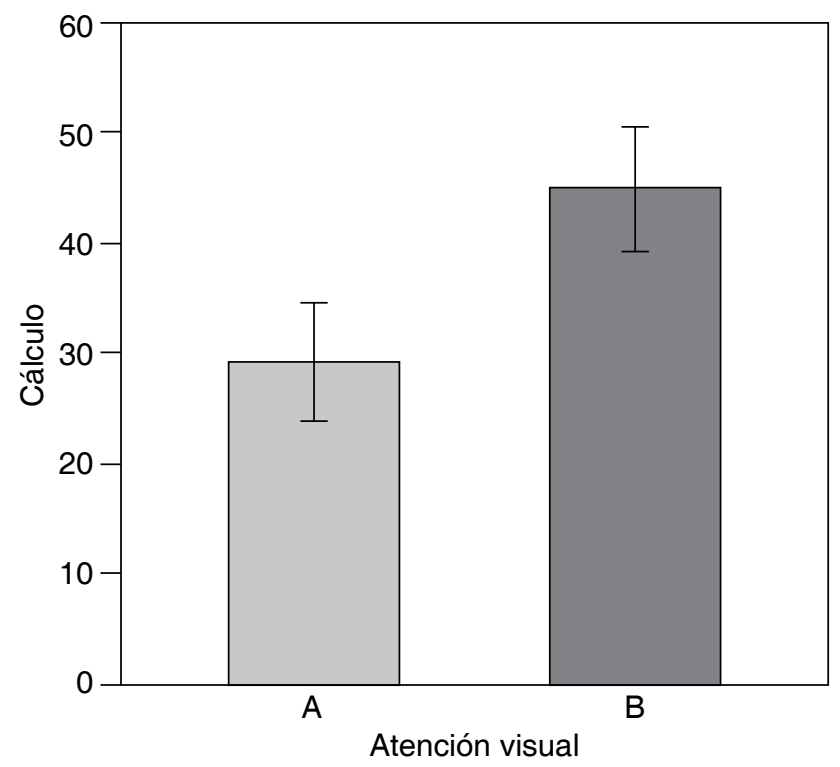

Figura 3. Comparación entre los puntajes de cálculo y atención visual. A corresponde a los puntajes bajos y $\mathrm{B}$ a los puntajes altos.

lo cual permitirá que el alumno universitario elija su carrera profesional por lo que le gusta hacer, independientemente de que incluya el estudio de las matemáticas. De esta forma se promoverán coyunturas favorables al conocimiento en diversas situaciones del ámbito personal, laboral y profesional. ${ }^{5}$

Habría que documentar las características de los métodos de enseñanza para corroborar si se promueve o no el desarrollo de la memoria y la atención visual en interacción con los diversos aprendizajes de la currícula en la educación básica, para que de esta forma se establezca si las dificultades en el aprendizaje de las matemáticas son resultado de alteraciones neurobiológicas o ambientales.

En esta investigación evaluamos a niños sin factores de riesgo biológico, de escuelas públicas, con diversos profesores y métodos de enseñanza. Sin embargo, habrá que contrastar los resultados con un grupo de niños de todos los grados escolares, con profesores que hagan actuar al alumno para que resuelva problemas, utilizando diversas estrategias de solución, reflexión, búsqueda y descubrimiento. De esta forma se logrará la construcción de significados. ${ }^{18}$

Diversos estudios han mostrado la activación de áreas parietales, frontales, prefrontales y de áreas subcorticales ante la solución de tareas matemáticas; a partir de esto se han propuesto metodologías para facilitar los procesos matemáticos partiendo de los conceptos concretos hasta llegar a los abstractos. De allí que debemos seguir investigando el desarrollo de las nociones lógico-matemáticas, las problemáticas que se están presentando en su aprendizaje y la implementación de métodos adecuados en todos los niveles educativos. 


\section{Conclusiones}

El mayor desempeño en las nociones matemáticas se observó en la resolución de problemas aritméticos y conteo, mientras que el menor desempeño se encontró en manejo numérico y cálculo.

Los niños con puntajes bajos en manejo numérico tuvieron puntajes bajos en memoria y habilidades espaciales. Los casos con puntajes bajos en conteo y cálculo tuvieron puntajes bajos en habilidades espaciales y atención visual, respectivamente.

\section{BIBLIOGRAFÍA}

1. Blanco L, Barona E, Carrasco A. Cognition and affect in mathematics problem solving with prospective teachers. The Mathematics Enthusiast. 2013; 10 (1-2): 334-364.

2. Abad D, Bocanegra Y, Giraldo C, González L. Caracterización neuropsicológica de los trastornos específicos del aprendizaje en una muestra de niños pereiranos. Revista Neuropsicología, Neuropsiquiatría y Neurociencias. 2012; 12 (2): 27-42.

3. Guiot M. Estudio de los comportamientos notacionales en niños preescolares (4 a 6 años) respecto al sistema de notación numérico convencional. Facultad de Psicología, Universidad Veracruzana-Xalapa. 2009; 5 (5): 1-21.

4. Piaget J. Psicología de la inteligencia. 2da. ed., Barcelona: Crítica, grupo Editorial Grijalbo. 1983.

5. Cardoso E, Cerecedo M. El desarrollo de las competencias matemáticas en la primera infancia. Revista Iberoamericana de Educación. 2008; 47 (5): 1-11.

6. Meneses S. Neurofisiología de la atención: potenciales relacionados a eventos. En: Alcaraz VM, Gumá E, editores. Texto de neurociencias cognitivas. México: Manual Moderno. $2001 ; 81-108$
7. Tang $\mathrm{Y}$, Posner M. Attention training and attention state training. Trends in Cognitive Sciences. 2009; 13 (5): 222-227.

8. Gumá E. La memoria humana. En: Alcaraz VM, Gumá E, editores. Texto de neurociencias cognitivas. México: Manual Moderno. 2001; 195-229.

9. Nunes T, Bryant P, Sylva K, Barros R. Development of math capabilities and confidence in primary school. Department of Education. London: University of Oxford. 2009; Research Report No. DCSF-RR118.

10. Piaget J. El juicio y el razonamiento en el niño. Estudio sobre la lógica del niño II. $3^{\underline{a}}$ ed., Buenos Aires: Editorial Guadalupe. 1992.

11. Dowker A. What works for children with mathematical difficulties? The effectiveness of intervention schemes. London: University of Oxford. 2009; 3: 1-52.

12. Dehaene S, Molko N, Cohen L, Wilson A. Arithmetic and the brain. Current Opinion in Neurobiology. 2004; 14: 218-224.

13. Gracia-Bafalluy M, Escolano-Pérez E. Aportaciones de la neurociencia al aprendizaje de las habilidades numéricas. Rev Neurol. 2014; 58 (2): 69-76.

14. Mundia L. The assessment of math learning difficulties in a primary grade- 4 child with high support needs: mixed methods approach. International Electronic Journal of Elementary Education. 2012; 4 (2): 347-366.

15. Geary D, Hoard M. Learning disabilities in arithmetic and mathematics: Theoretical and empirical perspectives. En: Campbell JID; editors. Handbook of mathematical cognition. New York: Psychology Press. 2005; 253-267.

16. Matute $\mathrm{E}$, Rosselli M, Ardila A, Ostrosky-Solís F. Evaluación neuropsicológica infantil (ENI). México: Manual Moderno. 2009.

17. Wechsler D. Escala de Wechsler de inteligencia para niñosIV. México: Manual Moderno. 2007.

18. Cobo P, Molina M. ¿Pueden nuestros estudiantes construir conocimientos matemáticos? Números. Revista Didáctica de las Matemáticas. 2014; 85: 49-73.

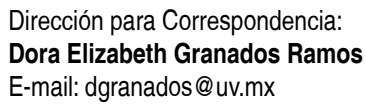

